ISSN 0103-9954

\title{
MODELAGEM DE MISTURAS NA FABRICAÇÃO DE COMPÓSITOS POLÍMERO-FIBRA, UTILIZANDO POLIETILENO E SERRAGEM DE Pinus sp.
}

\section{MODELLING OF COMPOSITIONS BASED ON WOOD-FIBER USING POLYETHYLENE AND Pinus sp. SAWDUST}

\section{Éverton Hillig ${ }^{2}$ Estevão Freire ${ }^{3}$ Gláucio A. Carvalho ${ }^{3}$ Vania Elisabete Schneider ${ }^{4}$ Karla Pocai ${ }^{5}$ \\ RESUMO}

Neste trabalho, foi estudado o efeito da composição de diferentes misturas de polietileno de alta densidade (HDPE) virgem, HDPE reciclado e serragem de Pinus sp., nas propriedades físico-mecânicas de placas confeccionadas pelo processo de compressão. As misturas foram homogeneizadas em um misturador tipo Drais, sem controle de temperatura e moldadas por compressão em prensa hidráulica a $150^{\circ} \mathrm{C}$. Partindo das placas, foram confeccionados corpos-de-prova para ensaios de tração, flexão, impacto e dureza, segundo normas ASTM, e também foram determinadas as densidades médias das placas. A modelagem estatística foi realizada segundo o planejamento centróide simplex, utilizando sete misturas dos três componentes e três repetições de cada mistura. Os resultados mostraram que a resistência à tração, a resistência à flexão, a dureza e a densidade das placas, são explicadas pelo modelo linear, enquanto a resistência ao impacto é explicada pelo modelo quadrático. Não houve diferença significativa nas propriedades físico-mecânicas dos compósitos confeccionados com HDPE virgem, daqueles confeccionados com HDPE reciclado, exceto para resistência ao impacto, no qual o HDPE virgem apresentou maiores valores.

Palavras-chave: compósitos; polietileno; serragem.

\section{ABSTRACT}

In this work, the effect of different compositions of virgin and recycled high density polyethylene/Pinus sp. on physical-mechanical properties of sheets made by compression molding was studied. The compositions were blended in a thermokinetic mixer (Draiss) and compression molded at $150^{\circ} \mathrm{C}$. ASTM samples for tensile, flexural, impact and hardness tests were taken from the sheets. The density of the sheets were determined. The statistical model used was a centroid simplex with seven compositions and three repetitions. Results showed that tensile and flexural strength as well as hardness and density followed a linear model, while impact strength is explained by a quadratic model. Physicalmechanical properties of compositions using virgin and recycled HDPE did not show significative changes, except for impact strength, when virgin HDPE showed higher numerical results.

Keywords: composites; polyethylene; sawdust.

\section{INTRODUÇÃO}

Segundo Rowell (1994), uma emergente preocupação ambiental no que diz respeito à reciclagem de materiais e os constantes aumentos do preço do petróleo tem incentivado a utilização de fibras naturais em compósitos. Tita (1999) considera um compósito como sendo qualquer material multifásico que exiba uma proporção significativa das propriedades de ambas as fases que o constituem, obtendo-se assim, uma melhor combinação de propriedades.

Nos compósitos poliméricos, geralmente o polímero é o componente contínuo, responsável pela transferência de um esforço sofrido para outro componente descontínuo que fornece a resistência ao esforço. A matriz polimérica, portanto, é o principal suporte constituinte que governa as propriedades mecânicas do material (Bittencourt, 2001). Novos compósitos, que podem oferecer benefícios sociais e ambientais, estão

1. Trabalho realizado com auxílio da Fapergs.

2. Engenheiro Florestal, MSc., Professor do Departamento de Engenharia e Informática, Universidade de Caxias do Sul, CEP 95700-000, Bento Gonçalves (RS). ehillig@ucs.br

3. Engenheiro Químico, MSc., Professor do Departamento de Engenharia Química, Universidade de Caxias do Sul, CEP 95010-550, Caxias do Sul (RS).

4. Bióloga, MSc., Professora do Departamento de Ciências Exatas e da Natureza, Universidade de Caxias do Sul, CEP 95700-000, Bento Gonçalves (RS).

5. Acadêmica do Curso de Graduação em Engenharia Química, Departamento de Engenharia Química, Universidade de Caxias do Sul, CEP 95010-550, Caxias do Sul (RS). Bolsista de Iniciação Científica.

Recebido para publicação em 9/03/2005 e aceito em 23/07/2006. 
sendo desenvolvidos para utilização nas indústrias automobilística, construção civil, móveis e embalagens.

Compósitos confeccionados com matriz polimérica, fibras de madeira e aditivos foram amplamente estudados por Song (2001). Constatou-se que diferentes materiais, com propriedades distintas, podem ser obtidos para as mais variadas aplicações utilizando métodos de processamento de plásticos tradicionais como extrusão, injeção e compressão. Segundo Jones (1998), a coloração escura que os compósitos eventualmente podem apresentar, é característica das fibras agregadas ao polímero e que, sozinhas, tendem a degradar rapidamente na exposição à luz solar e ao ataque de microorganismos.

Diversos tipos de fibras naturais têm sido utilizados e se constituem basicamente de celulose e hemicelulose, com moléculas unidas umas às outras por lignina. Esses constituintes, por sua vez, são polímeros naturais que podem substituir os polímeros sintéticos em compósitos, possibilitando o desenvolvimento de novos produtos e novas tecnologias de produção (Oashi, 1999; Sanadi et al, 1997). Sanadi et al. (1998) destacam que, dos materiais utilizados como fibras naturais em compósitos, a fibra de madeira representa um bom potencial, em conseqüência da elevada geração de resíduos quando de seu processamento na indústria madeireira.

Tratando-se de termoplásticos, Pacheco (2000) comenta que o polietileno de alta densidade (HDPE) é bastante utilizado, em razão da facilidade de moldagem e grande aplicação na fabricação de embalagens. Por outro lado, Harper (1996) demonstrou que, quando reciclado apropriadamente, esse polímero mantém razoavelmente bem as propriedades mecânicas e de fluxo, podendo ter, entretanto, a resistência ao impacto e à degradação ambiental diminuídas.

Outro fator a considerar na confecção de compósitos utilizando materiais termoplásticos e lignocelulósicos é a temperatura de fluidez do termoplástico que precisa estar abaixo da temperatura de degradação do material lignocelulósico, situada geralmente entre 200 e $220^{\circ} \mathrm{C}$. Apesar das fibras causarem aumento da rigidez e da resistência ao impacto do produto, sua baixa resistência ao calor deverá orientar na seleção correta do material polimérico a ser utilizado (Youngquist, 1999).

Os materiais lignocelulósicos apresentam vantagens quando usados em compósitos com termoplásticos, pois, comparando-os com os materiais inorgânicos, são recursos renováveis que possuem menor densidade e baixo custo. Em contrapartida, por causa da sua característica polar, não dispersam facilmente na matriz polimérica (Oashi, 1999; Maldas e Kokta, 1993).

Com a finalidade de melhorar a interação entre a fibra na forma de serragem e o polímero, é importante realizar um pré-tratamento das fibras ou utilizar agentes de acoplamento que possibilitem a formação de um compósito homogêneo (Bataille et al., 1990).

As propriedades físico-mecânicas também podem ser melhoradas com o uso de agentes que promovam a adesão interfacial das fibras da madeira com o polímero. De acordo com Gauthier et al. (1998), os agentes compatibilizantes mais eficientes devem possuir uma função química altamente reativa com os grupos OH da celulose. Segundo Maldas e Kokta (1993) e Data e Lohse (1996) os mais utilizados são os isocianatos, anidridos e silanos. No entanto, dentre os anidridos, o que mais se destaca na graftização de poliolefinas é o anidrido maleico, por promover ligações covalentes entre o polímero e as hidroxilas da superfície das fibras (Rowell et al., 1997). Essa interface formada é extremamente importante para o bom desempenho mecânico e integridade estrutural dos compósitos, pois, mesmo com a grande diversidade de resinas existentes, estas geralmente não resistem às tensões que as fibras suportam, sobretudo considerando a resistência à flexão (Silvestre, 2001).

Outros fatores, como tipo e forma da fibra, também podem ser responsáveis por mudanças nas propriedades mecânicas dos compósitos confeccionados. Trabalhando com diferentes procedências e formas de fibras de madeira em compósitos com 30\% de fibras e 70\% de polipropileno, Mahlberg et al. (2001) concluíram que a aparência e as propriedades mecânicas dos compósitos são influenciadas pela origem, forma e tamanho das partículas de madeira utilizadas.

Verifica-se, assim, que a fase dispersa nos compósitos confeccionados adquire maior importância, passando de simples carga adicionada para constituinte ativo de uma mistura e que tem influência direta nas suas propriedades fisico-mecânicas. 
Para estudar o efeito da composição de uma mistura na variável resposta de um ensaio, pode-se utilizar a modelagem de misturas. Nesse caso, segundo Neto et al. (2001), duas considerações são importantes: as propriedades de uma mistura são determinadas pelas proporções de seus componentes e as proporções dos diversos componentes são dependentes. Em geral, para a modelagem de misturas, podem ser utilizados modelos lineares, quadráticos ou cúbicos que usam o mesmo número de ensaios que o número de coeficientes que se quer estimar. Esse método, conhecido como "planejamento em rede simplex", é utilizado especialmente para misturas de três componentes. Nesse caso, em razão da particularidade das misturas ternárias serem representadas por apenas uma mistura, o planejamento é conhecido como "centróide simplex".

Tendo por base essas informações, foi realizado o presente estudo que visou a utilizar resíduos (serragem) das indústrias moveleira e madeireira da serra gaúcha, incorporando-os ao polietileno de alta densidade (HDPE), virgem e reciclado. Formulações de serragem/polímeros foram usadas para compor as misturas que, com a adição de um agente de acoplamento, foram avaliadas por meio de suas propriedades físico-mecânicas e de acordo com o planejamento experimental centróide simplex.

\section{MATERIAL E MÉTODOS}

\section{Confecção das placas}

Para confecção das placas, a mistura foi homogeneizada em um misturador tipo Drais, sem controle de temperatura. Após processado, o material foi colocado em prensa quente, marca Schulz PHS com capacidade de 15 toneladas, num molde com dimensões de $150 \times 150 \times 3 \mathrm{~mm}$. A temperatura da prensa foi ajustada em $150^{\circ} \mathrm{C}$, o tempo de prensagem fixado em 5 minutos e a força aplicada foi de 7 toneladas, seguindo-se o resfriamento em prensa fria com força de 7 toneladas.

Posteriormente, foram confeccionados corpos de prova de cada chapa para realização de ensaios de resistência à tração, ao impacto, à flexão e de determinação da dureza Shore $\mathrm{D}$, seguindo as recomendações das normas ASTM D638, D256, D790 e D2240 respectivamente.

\section{Análise dos resultados}

O modelo experimental utilizado é conhecido como planejamento em rede centróide simplex. Nesse delineamento, o modelo mais simples para uma mistura é o modelo linear ou aditivo, que procura explicar o comportamento de uma propriedade apenas com os resultados obtidos com a utilização de cada componente individualmente, isto é, prever o comportamento de qualquer mistura sem realizar nenhuma mistura. Numa mistura de três componentes, pode-se ter, além deste, modelos quadráticos que consideram os efeitos das interações de dois componentes e modelos cúbicos que consideram os efeitos das interações dos três componentes.

Os modelos utilizados são expressos nas equações derivadas 1, 2 e 3 respectivamente.

$$
\begin{aligned}
& Y_{i}=b_{1} \cdot X_{1}+b_{2} \cdot X_{2}+b_{3} \cdot X_{3} \\
& Y_{i}=b_{1} \cdot X_{1}+b_{2} \cdot X_{2}+b_{3} \cdot X_{3}+b_{12} \cdot X_{1} \cdot X_{2}+b_{13} \cdot X_{1} \cdot X_{3}+b_{23} \cdot X_{2} \cdot X_{3} \\
& Y_{i}=b_{1} \cdot X_{1}+b_{2} \cdot X_{2}+b_{3} \cdot X_{3}+b_{12} \cdot X_{1} \cdot X_{2}+b_{13} \cdot X_{1} \cdot X_{3}+b_{23} \cdot X_{2} \cdot X_{3}+b_{123} \cdot X_{1} \cdot X_{2} \cdot X_{3} \\
& \quad \text { Em que: } Y i=\text { Variável resposta; } b i=\text { Coeficientes } X i=\text { Proporção de cada espécie na mistura. }
\end{aligned}
$$

Para a determinação dos coeficientes, foram utilizadas as sete misturas mínimas necessárias para o modelo cúbico. As misturas utilizadas são apresentadas na Tabela 1, destacando que, para cada mistura, foram realizadas três repetições. Como não é possível fabricar uma chapa apenas com serragem, a mistura 7 contém o máximo possível desse material, sendo um pseudo-componente que visa a representar a utilização de serragem pura.

$\mathrm{Na}$ avaliação dos modelos, foram testados modelos cúbicos para todas as propriedades analisadas, realizada análise da variância, e descartados os coeficientes não-significativos pelo teste "t". Para derivação dos coeficientes, utilizou-se o software SPSS. 
TABELA 1: Composições utilizadas para a os testes mecânicos.

TABLE 1: Compositions used for the mechanical tests.

\begin{tabular}{c|cccc}
\hline Placa & HDPE virgem (\%) & Serragem (\%) & HDPE reciclado (\%) \\
\hline 1 & 100 & 0 & 0 \\
2 & 50 & 0 & 50 \\
3 & 0 & 0 & 100 \\
4 & 33,3 & 33,3 & 33,3 \\
5 & 0 & 50 & 50 \\
6 & 50 & 50 & 0 \\
7 & 12,5 & 75 & 12,5 \\
\hline
\end{tabular}

Em que: $\mathrm{HDPE}=$ polietileno de alta densidade.

\section{RESULTADOS E DISCUSSÃO}

A Tabela 2 apresenta os resultados médios das propriedades físico-mecânicas dos compósitos confeccionados com as diferentes misturas de HDPE virgem, HDPE reciclado e serragem.

TABELA 2: Resultados médios dos ensaios fisico-mecânicos dos corpos de prova confeccionados.

TABLE 2: Mean values of physical-mechanical tests of samples.

\begin{tabular}{c|c|c|c|c|c|c|c|c}
\hline Placa & $\begin{array}{c}\text { HDPE } \\
\text { Virgem } \\
(\%)\end{array}$ & $\begin{array}{c}\text { Serragem } \\
(\%)\end{array}$ & $\begin{array}{c}\text { HDPE } \\
\text { reciclado } \\
(\%)\end{array}$ & $\begin{array}{c}\text { Resistência à } \\
\text { tração }(\mathrm{MPa})\end{array}$ & $\begin{array}{c}\text { Resistência à } \\
\text { flexão }(\mathrm{MPa})\end{array}$ & $\begin{array}{c}\text { Resistência } \\
\text { ao Impacto } \\
(\mathrm{J} / \mathrm{m})\end{array}$ & $\begin{array}{c}\text { Densidade } \\
\left(\mathrm{g} / \mathrm{cm}^{3}\right)\end{array}$ & Dureza \\
\hline 1 & 100 & 0 & 0 & 24,73 & 24,87 & 38,34 & 0,97 & 65 \\
2 & 50 & 0 & 50 & 25,40 & 24,77 & 30,92 & 0,97 & 65 \\
3 & 0 & 0 & 100 & 25,66 & 24,87 & 33,90 & 0,96 & 64 \\
4 & 33,3 & 33,3 & 33,3 & 21,79 & 31,11 & 23,03 & 1,07 & 69 \\
5 & 0 & 50 & 50 & 17,78 & 33,43 & 21,82 & 1,11 & 73 \\
6 & 50 & 50 & 0 & 16,70 & 29,26 & 20,77 & 1,12 & 73 \\
7 & 12,5 & 75 & 12,5 & 16,76 & 33,19 & 15,63 & 1,21 & 75 \\
\hline
\end{tabular}

Em que: $\mathrm{HDPE}=$ polietileno de alta densidade.

Na Tabela 3, são apresentadas as equações simplificadas, nas quais foram descartados os coeficientes não-significativos pelo teste " $t$ " de student, para as propriedades fisico-mecânicas das placas.

TABELA 3: Equações simplificadas para a densidade e propriedades mecânicas das chapas, utilizando-se apenas os coeficientes significativos pelo teste " $t$ ".

TABLE 3: Simplified equations for density and mechanical properties of sheets, using only the significative coefficients taken from test " $t$ ".

\begin{tabular}{|c|c|c|c|}
\hline Propriedade & Equação & $\mathrm{F}$ & $\mathrm{R}^{2}$ \\
\hline Densidade & Densidade $=0,969 * \mathrm{PEV}+0,959 * \mathrm{PER}+1,316 * \mathrm{SER}$ & $116,57^{1}$ & 0,980 \\
\hline RT & Tração $=24,73 * \mathrm{PEV}+25,66 * \mathrm{PER}+16,36 * \mathrm{SER}$ & $15,19^{1}$ & 0,867 \\
\hline $\mathrm{RF}$ & Flexão $=24,87 * \mathrm{PEV}+24,87 * \mathrm{PER}+32,90 *$ SER & $7,02^{1}$ & 0,750 \\
\hline RI & Impacto $=38,34 * \mathrm{PEV}+33,90 * \mathrm{PER}+12,20 * \mathrm{SER}-20,81 * \mathrm{PEV} * \mathrm{PER}$ & $35,05^{1}$ & 0,937 \\
\hline Dureza & Dureza $=65,00 * \mathrm{PEV}+64,33 * \mathrm{PER}+76,88 * \mathrm{SER}$ & $12,50^{1}$ & 0,843 \\
\hline
\end{tabular}

Em que: 1 = significativo ao nível de $5 \%$ de probabilidade de erro; $\mathrm{F}=$ valor de $\mathrm{F}$ calculado; $\mathrm{R}^{2}=$ coeficiente de determinação; PEV = Proporção de polietileno de alta densidade virgem; PER = Proporção de polietileno de alta densidade reciclado; $\mathrm{RT}=$ Resistência à Tração; $\mathrm{RF}=$ Resistência à Flexão; RI = Resistência ao Impacto; SER = Proporção de serragem.

A análise dos resultados permitiu verificar que a resistência à tração diminui com o aumento do teor de serragem. Na equação simplificada, o coeficiente para a proporção de serragem tem valor menor que os coeficientes derivados para o HDPE virgem e HDPE reciclado, indicando uma diminuição dessa propriedade em aproximadamente $35 \%$ com a adição de serragem. Por outro lado, a semelhança entre os valores dos coeficientes do HDPE virgem e do reciclado, sendo esse último ligeiramente superior, mostram que a reciclagem não prejudica as propriedades mecânicas do polímero. Esses resultados confirmam as afirmações de Harper (1996), de que o polietileno quando reciclado mantém suas propriedades mecânicas. 
A Figura 1 apresenta o gráfico ternário da resistência à tração em razão da mistura dos três componentes. Pode ser observado que o polietileno reciclado possui boas propriedades, pois as linhas de resistência à tração não são paralelas ao vértice do HDPE virgem e mostram uma pequena diminuição dessa propriedade à medida que aumenta a proporção de HDPE virgem em relação ao HDPE reciclado.

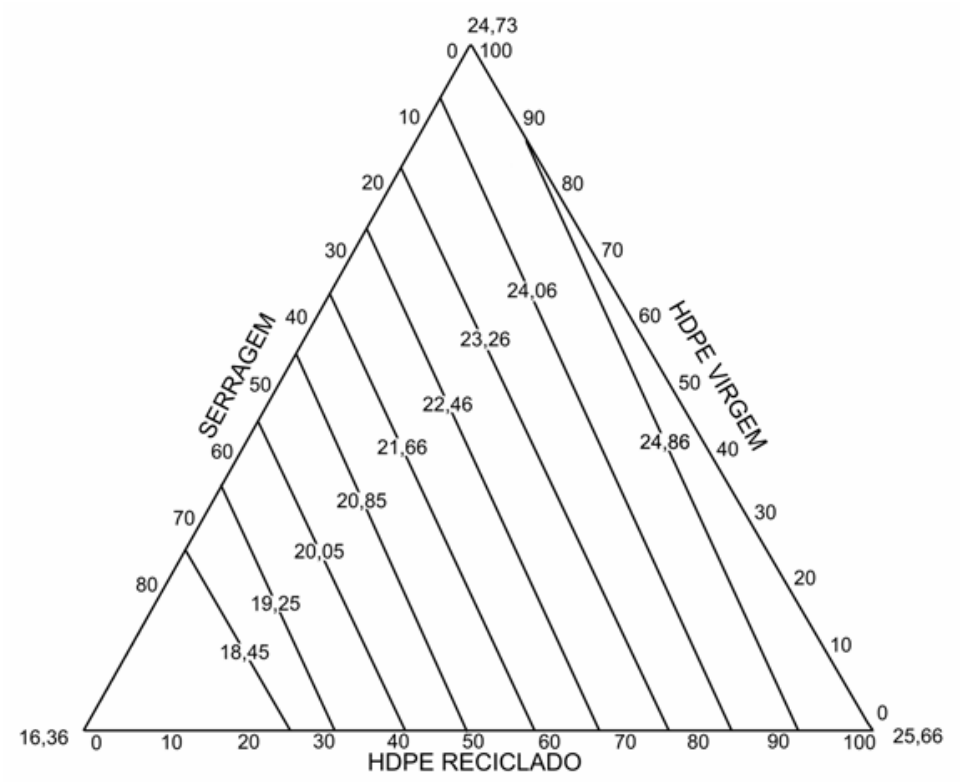

FIGURA 1: Gráfico ternário da resistência à tração dos compósitos em função da mistura de HDPE virgem, HDPE (polietileno de alta densidade) reciclado e serragem.

FIGURE 1: Ternary plot of tensile strength of composites as a function of virgin HDPE, recycled HDPE and sawdust content.

Para a resistência à flexão, os coeficientes também foram semelhantes para o HDPE virgem e o reciclado. A adição de serragem provocou melhora na resistência à flexão, fato também constatado por Youngquist (1999) em seu trabalho, e confirmado pelo valor do coeficiente encontrado para serragem, igual a 32,90. Esse efeito era esperado, pois as fibras da madeira possuem altos valores de resistência à flexão, superiores aos apresentados pelo polietileno. Não houve nenhum tipo de interação, binária ou ternária, entre as misturas estudadas, permitindo concluir que a resistência à flexão, da mesma forma que a resistência à tração, é afetada apenas pelas características individuais de cada componente da mistura. A Figura 2 apresenta o gráfico ternário de resistência à flexão em função da mistura dos três componentes. Nesse caso, verifica-se, pelas linhas paralelas ao vértice do HDPE virgem, que não houve diferenças entre esse e o HDPE reciclado. 


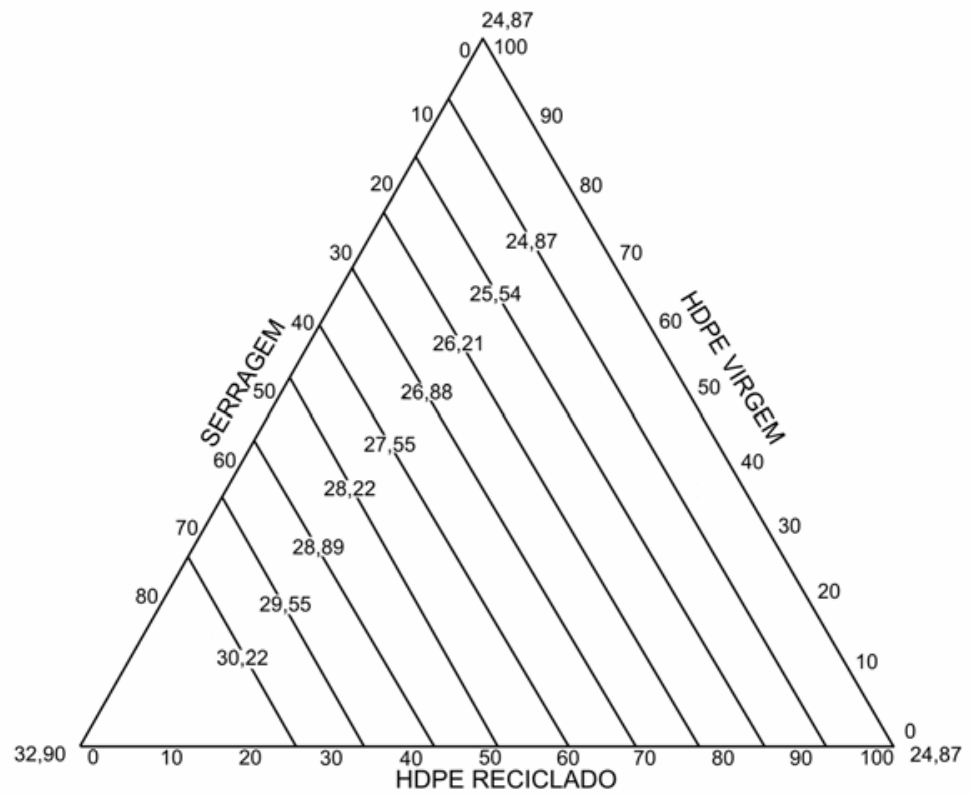

FIGURA 2: Gráfico ternário da resistência à flexão dos compósitos em função da mistura de HDPE virgem, HDPE (polietileno de alta densidade) reciclado e serragem.

FIGURE 2: Ternary plot of flexural strength of composites as a function of virgin HDPE, recycled HDPE and sawdust content.

A adição de serragem causou diminuição dos valores de resistência ao impacto e aumento dos valores de dureza, sendo o efeito mais pronunciado para a resistência ao impacto. Nesse caso, o coeficiente linear calculado para o polietileno reciclado foi um pouco inferior ao calculado para polietileno virgem, confirmando as considerações de Harper (1996) quando comenta que a resistência ao impacto do polietileno reciclado pode sofrer diminuição quando submetido à reciclagem. Verificou-se, ainda, que o polietileno virgem e o polietileno reciclado interagiram antagonisticamente, pois o coeficiente calculado para a interação é negativo. Esse fato pode ser atribuído à diferença de polaridade entre a resina virgem e a reciclada, ocasionada pela degradação e conseqüente aumento da polaridade da resina reciclada.

A Figura 3 apresenta os gráficos ternários de resistência ao impacto e de dureza, em função da mistura dos três componentes. Pode-se observar que, pela interação entre HDPE virgem e HDPE reciclado, o gráfico de impacto apresenta as linhas curvas, com tendência de diminuição dos valores à medida que aumentam as proporções da mistura dos polietilenos.

Por fim, o aumento da proporção de serragem causou aumento da densidade do compósito. Esse fato já foi verificado por Hillig et al. (2002), em conseqüência da compactação da serragem no momento da prensagem. Tendo em vista que a densidade da madeira utilizada no presente estudo é de aproximadamente $0,5 \mathrm{~g} / \mathrm{cm}^{3}$, o coeficiente linear da serragem calculado para a densidade igual a 1,316 mostra que ocorreu compactação das fibras de madeira provocada pela força aplicada. A Figura 4 apresenta o gráfico ternário da densidade dos compósitos em função da mistura dos três componentes. 

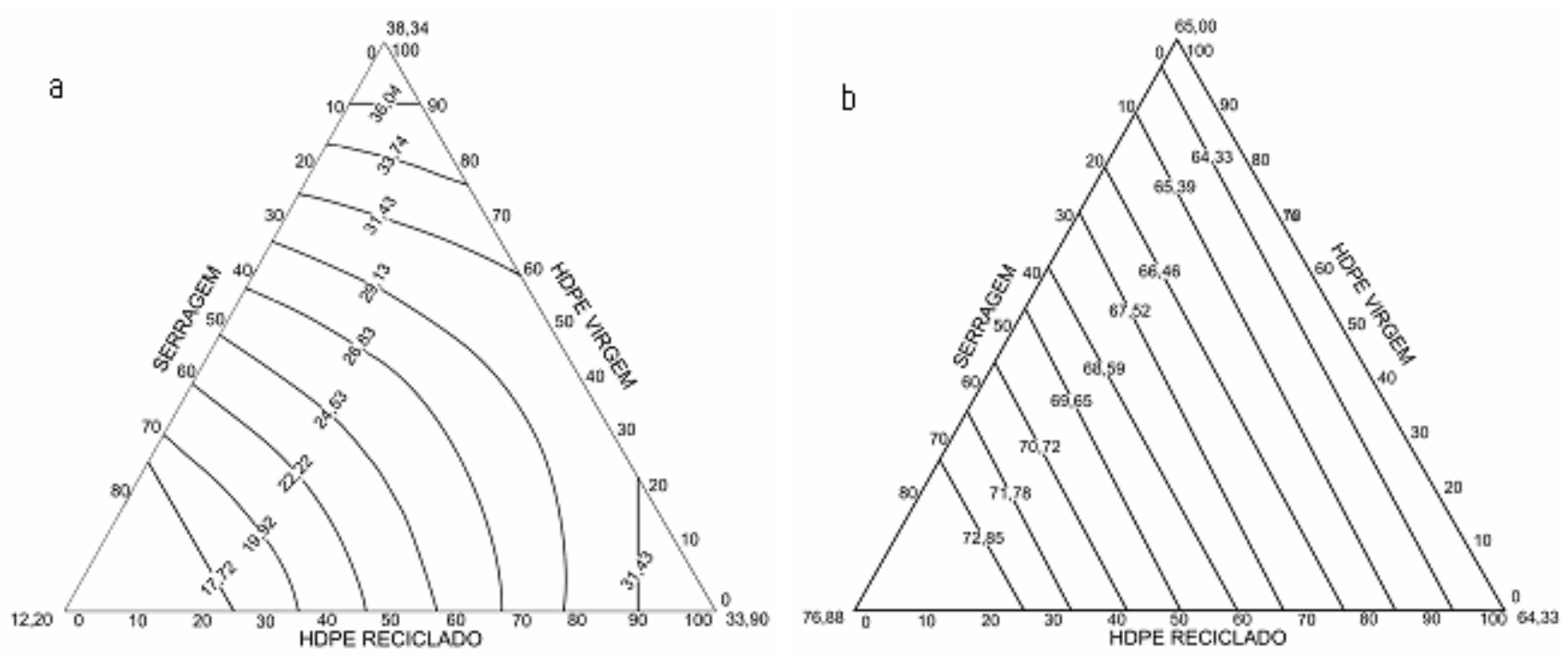

FIGURA 3: Resistência ao impacto (a) e dureza (b) em função da mistura de HDPE (polietileno de alta densidade) virgem, HDPE reciclado e serragem.

FIGURE 3: Impact strength (a) and hardness (b) of composites as a function of virgin HDPE, recycled HDPE and sawdust content.

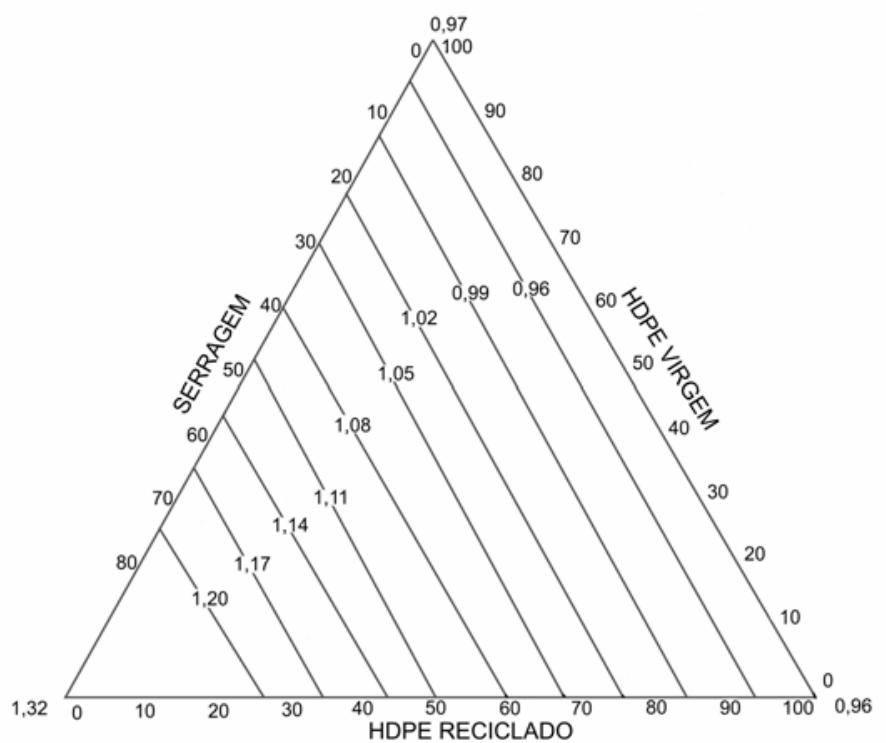

FIGURA 4: Gráfico ternário da densidade dos compósitos em função da mistura de HDPE virgem, HDPE reciclado e serragem.

FIGURE 4: Ternary plot of density of composites as a function of virgin HDPE, recycled HDPE and sawdust content.

\section{CONCLUSÕES}

Não houve diferença significativa nas propriedades físico-mecânicas dos compósitos confeccionados com HDPE virgem, em relação aos confeccionados com HDPE reciclado, exceto para resistência ao impacto, na qual o HDPE virgem apresentou maiores valores. Todos os compósitos confeccionados apresentaram boas propriedades físico-mecânicas, atestando a viabilidade de aplicação industrial.

A modelagem de misturas mostrou que a resistência à tração, a resistência à flexão, a dureza e a densidade das chapas são determinadas pelo modelo linear, ou seja, são influenciadas apenas pelas características individuais de cada componente na mistura. Por outro lado, na resistência ao impacto, os polietilenos, virgem e reciclado, interagiram antagonisticamente, ou seja, provocando diminuição dessa 
propriedade. Dessa forma, as propriedades que seguiram o modelo linear podem ser previstas por meio da soma das propriedades individuais de cada componente da mistura, enquanto a resistência ao impacto deve levar em consideração a interação entre HDPE virgem e HDPE reciclado.

\section{AGRADECIMENTOS}

Os autores agradecem à Fundação de Amparo à Pesquisa do Estado do Rio Grande do Sul FAPERGS e à empresa Peretto Materiais de Construção Ltda, pelo auxílio financeiro. Também ao bolsista Otavio Bianchi pela confecção dos gráficos.

\section{REFERÊNCIAS BIBLIOGRÁFICAS}

AMERICAN SOCIETY FOR TESTING AND MATERIALS. ASTM STANDARD D256-97 : standard test methods for detemining the izod pendulum impact resistance of plastics. West Conshohocken: ASTM, 2000. (Annual book of ASTM standards ; v. 08.01)

AMERICAN SOCIETY FOR TESTING AND MATERIALS. ASTM STANDARD D638-99 : standard test method for tensile properties of plastics. West Conshohocken: ASTM, 2000. (Annual book of ASTM standards ; v. 08.01)

AMERICAN SOCIETY FOR TESTING AND MATERIALS. ASTM STANDARD D790-99 : standard test method for flexural properties of unreinforced and reinforced plastics and electrical insulating materials. West Conshohocken: ASTM, 2000. (Annual book of ASTM standards ; v. 08.01)

AMERICAN SOCIETY FOR TESTING AND MATERIALS. ASTM STANDARD D 2240-97 : standard test method for rubber property - durometer hardness. West Conshohocken: ASTM, 2000. (Annual book of ASTM standards ; v. 09.01)

BATAILLE P.; ALLARD P.; COUSIN, P.; SAPIEHA, S. Interfacial phenomena in cellulose/polyethylene composites. Polymer Composites, v. 11, n. 5, p. 301-304, 1990.

BITTENCOURT, C. B. Procedimento de ensaio para avaliar propriedades ao cisalhamento de materiais compósitos : método da viga com entalhe em V. Itajubá, 2001. 14f. Monografia (Conclusão de Curso de Engenharia) Escola Federal de Engenharia de Itajubá, Itajubá, 2001.

DATA, S.; LOHSE, D. J., Polymeric compatibilizers uses and benefits in polymer blends. Ohio: Hanser/Gardner Publications, 1996. 542p.

GAUTHIER, R.; JOLY, C.; COUPAS, A. C.; GAUTHIER, H.; ESCOUBES, M. Interfaces in polyolefin/cellulosic fiber composites: chemical coupling, morphology correlation with adhesion aging in moisture. Polymer Composites, v. 69 , p. $2195-2203,1998$.

HARPER, C. A. Handbook of plastics, elastomers and composites. 3rd ed. New York : McGraw-Hill, 1996. 677p.

HILLIG, É.; AGUZZOLI, C.; FREIRE, E.; MANSAN, M. A. Estudo de diferentes granulometrias e proporções de resíduos de madeira (serragem) misturados com PEDB. In: CONGRESSO EM CIÊNCIAS DE MATERIAIS DO MERCOSUL-SULMAT, 2002, Joinville. Anais... Joinville : Tec Art Editora, 2002. p. 308-315.

JONES, R. F. Guide to short fiber reinforced plastics. Cincinnati : Hanser/Gardner, 1998. 172p.

MAHLBERG, R.; LAINE, A.; SUOMI-LINDBERG, L.; KIVISTÖ, A.; AHOLA, P. Effect of fiber type and fiber modification on moisture behavior and mechanical properties of woodfiber-polypropylene composites. In: INTERNATIONAL CONFERENCE ON WOODFIBER-PLASTIC COMPOSITES, 6. , 2001, Madison. Proceedings... Madison : USDA Forest Service, 2001. Resumo disponível em: <www.forestprod.org/wfpl01abs.pdf $>$. Acesso em 2004.

MALDAS, D.; KOKTA, B. V. Role of coupling agents and treatments on the performance of wood fiber-thermoplastic composites. In: Wolcott, M. P. (Ed.) Wood and fiber polymer composites : fundamental concepts, processes, and material options. Madison: Forest Products Society, 1993. p. 113-120.

NETO, B. B.; SCARMINIO, I. S.; BRUNS, R.E. Como fazer experimentos: pesquisa e desenvolvimento na ciência e na indústria. Campinas: Ed. da Unicamp, 2001. 401p.

OASHI, M. C. G. Estudo da cadeia produtiva como subsídio para pesquisa e desenvolvimento do agronegócio do sisal na Paraíba. Florianópolis, 1999. 177f. Tese (Doutorado em Engenharia de Produção) - Universidade Federal de Santa Catarina, Florianópolis, 1999.

PACHECO, E. B. Análise de impacto ambiental devido a resíduos poliméricos. Plástico Moderno, n. 308, p. 40-51, 2000. 
ROWELL, R. M. Utilization of recycled agriculture-based fiber for composites. In: RADER, C. P.; BALDWIN, S. D.; CORNELL, D. D. [and others], (Ed.) Plastics, rubber, and paper recycling : a pragmatic approach. NATIONAL MEETING AMERICAN CHEMICAL SOCIETY, 208., 1994. Proceedings... Washington: American Chemical Society, 1994. p. 357-366. Chap. 29. (ACS symposium series 609)

ROWELL, R. M.; SANADI, A. R.; CAUFIELD, D. F.; JACOBSON, R. E. Utilization of natural fibers in plastic composites: problems and opportunities. Wisconsin: Forest Products Laboratory, ESDA, Department of Forestry, University of Wisconsin, 1997.

SANADI, A. R.; FENG, D.; CAULFIELD, D. F. Highly filled lignocellulosic reinforced thermoplastics: effect of interphase modification. In: RISO INTERNATIONAL SYMPOSIUM ON MATERIALS SCIENCE: POLYMERIC COMPOSITES - EXPANDING THE LIMITS, 18., 1997, Roskilde. Proceedings... Roskilde: Riso National Laboratory, 1997. p. 465-470.

SANADI, A.; CAUFIELD, D. F.; ROWELL, R. M. Lignocellulosic plastic composites. Technology Summaries. Disponível em: <www.fpl.fs.fed.us/documnts/pdf1998/sanad98a.pdf>. Acesso em: dez. 2004.

SILVESTRE FILHO, G. D. Comportamento mecânico do poliuretano derivado de óleo de mamona reforçado por fibra de carbono: contribuição para o projeto de hastes de implante de quadril. São Paulo, 2001. 192f. Dissertação (Mestrado) - Universidade de São Paulo, 2001.

SONG, W. Study on the properties of various woodfiber-plastic formulations. In: INTERNATIONAL CONFERENCE ON WOODFIBER-PLASTIC COMPOSITES, 6., 2001, Madison. Proceedings... Madison: USDA Forest Service, 2001. Resumo disponível em: <www.forestprod.org/wfpl01abs.pdf>. Acesso em dezembro de 2004.

TITA, V. Análise dinâmica e experimental de vigas fabricadas a partir de materiais compósitos poliméricos reforçados. São Carlos, 1999. 155f. Dissertação (Mestrado em Engenharia Mecânica) - Universidade de São Paulo, Escola de Engenharia, São Carlos, 1999.

YOUNGQUIST, J. A. Wood-based composites and panel products. Wood handbook: wood as an engineering material. Washington: Forest Products Society, 1999. 428p. 